\title{
Histopathological and Epidemiological Analysis of cancer patients at a Tertiary Care Hospital
}

\author{
Dr. G.Sudhakar ${ }^{1}, *$ Dr. T.Rayapa Reddy ${ }^{2}$ \\ ${ }^{I}$ (Associate Professor, Department of Pathology, Guntur Medical College,NTR UHS, India) \\ ${ }_{2}^{2}$ (Assistant Professor, Department of Pathology, Guntur Medical College,NTR UHS, India) \\ *Corresponding author: Dr. T.Rayapa Reddy2
}

\begin{abstract}
Cancer incidence has been increasing throughout world including India in the recent years. The present study is focused on studying epidemiology of cancer in tertiary care hospital in south India. This is retrospective study conducted at Government general Hospital, Guntur, and Andhra Pradesh, India from January 2015 to December 2016. We analyzed the patients admitted during this study period, all possible information regarding age, gender, demographic data, cancer type, site and stage wise cancer, treatment, follow up period were gathered and analyzed statistically. A total of 236 patients, of mean age 49.45 years (SD: 11.27), including $52.33 \%$ male and $47.67 \%$ female were admitted during the study period. The most predominant age group was $41-60$ years. Among male, tongue cancer (11.92\%) and among female breast cancer were more predominant (17.86\%). The sub types of cancers, histopathological variants, stage wise presentation, treatment administered and follow up periods were explained in detail .This study explains the proportion of various cancers cases in south Indian population and provides a source of information, which help public health planners, administrators, healthcare system, and general public in the primary prevention and early detection of cancer which helps in detecting the cancer early.
\end{abstract}

Keywords: Histopathology, Prevalence, Prevention, Treatment

\section{Introduction}

Cancer occupies second position in the causes of mortality in developing as well as developed countries. [1] Incidence of cancer has been rapidly increasing throughout world in the recent years. There exists a wide variety of reasons behind this including tobacco, alcohol consumption, dietary habits and behavioral factors exposure to pesticides and chemicals, ionizing radiation, infection, hormonal imbalance, decreased immunity, heredity, etc. use of Tobacco, either by way of chewing or smoking accounts for $50 \%$ of all cancers in men, sexual practices and dietary practices account for $20-30 \%$ of cancers . It is estimated that around 2.5 million, with over 8,00,000 new cases and 5,50,000 deaths occurring due to cancer each year in India.[2] India, having multi social habitats and cultural shows that prevalence of cancer different from one area to other. The incidence of gall bladder cancers are more in Northern India, particularly in Delhi and West Bengal, pharyngeal cancers are more in Western India (Mumbai), stomach and Esophageal cancers is more in Southern India.[3,4] Clinical Pharmacology and Clinical epidemiology are two essential areas for integrating the health sciences as well as a source for future trends. From the fast 50 years, larger efforts have been spent for gathering data across hospital, state, countries as to study the impact of cultural factors and environmental on cancer incidence. The past epidemiological reports of cancer have shown that illiteracy and poverty are the major factors for the deaths from oral as well as cervical cancers. As higher cancer prevalence present in Guntur district in Andhra Pradesh, South India. We focused to study the cancer epidemiology in this particular area basing on the cancer registries of this tertiary care hospital.

\section{Materials And Methods}

In order to assess the differences in risk among populations require incidence rates, of which the data is derived from population based cancer registries, which aim to record information on all newly occurring cases in a particular population. We collected and analyzed data of all patients who were admitted with any type of cancer to Government general hospital, Guntur from January 2015 to December 2016. It is an 1100 bedded government tertiary care teaching hospital in Guntur, Andhra Pradesh, India. It is the referral hospital for cancer patients from Guntur district, other districts like Krishna, Prakasam, Nellore and Kadapa. Total In-patient admissions to the hospital average about 300-350 per day. Present data were gathered retrospectively from medical record case sheets. All hospitalized cancer patients were included. For the patients admitted during this study period, all possible information, gender, regarding age, demographic data, type of cancer, site -wise and stage-wise distribution of cancer, mode of treatment, follow-up period were gathered. Later data from all case sheets were analyzed statistically. 


\section{Results And Discussion}

Incidence of cancer has been rapidly increasing in recent times. This is mainly because of lifestyle changes, urbanization, and industrialization, exposure to pesticides, population growth and increased life span. In India, the life expectancy has steadily risen from 1971 (45 years) to 1991 (62 years), which indicates a shift in the socio-demographic profile.[5] Guntur district in Andhra Pradesh, Southern India, is having a large population and many cancer cases each year. Previous studies in this area have shown that the incidence is getting increased [6]. In our present study totally 236 patients were admitted with the diagnosis of cancer. Among them $52.33 \%$ male and $47.67 \%$ female with a mean age of 49.45 years (SD: 11.27). Men outnumbered women in all age groups, except the age group 21-40 years. The most predominant age group was 41-60 years. This shows the increased life expectancy.[5] Nearly half of the patients (48.51\%) were aged in between $41-60$ years and there were very few cases in the age groups $0-20$ years and $>80$ years. This data is indicated in Table no: 1 . Most of the patients were from rural background (88.93) and very few of them were from urban areas $(11.06 \%)$ as this area is having more rural population. In recent days exposure of agricultural workers to a variety of physical, chemical and biological hazards in the process of cultivating and harvesting crops and/or raising livestock is getting increased $[7,8]$.

AGE GROUP (Yrs)
$0-20$
$21-40$
$(11.06 \%)$
$41-60$
$(22.97 \%)$
$61-80$
$(13.61 \%)$
$>80$
TOTAL
$(47.65 \%)$

Table 1: Age -Sex distribution

$\begin{array}{ccc}\text { TOTAL } & \text { MALE } & \text { EEMALE } \\ 1(0.42 \%) & 1(0.42 \%) & 0 \\ 44(18.72 \%) & 18(7.65 \%) & 26 \\ & & \\ 114(48.51 \%) & 60(25.53 \%) & 54 \\ 74(31.48 \%) & 42(17.87 \%) & 32 \\ & & \\ 2(0.85 \%) & 2(0.85 \%) & 0 \\ 235(100 \%) & 123(52.34 \%) & 112\end{array}$

Due to bad habits such as chewing of betel nut, tobacco consumption in the form of chewing or smoking, mouth cancers are common in India. Reverse smoking also causes cancer of hard palate which is also known as reverse chutta cancer. [9] In our study Head and neck cancers are the major type of cancer (42\%), including $22.51 \%$ oral cavity and $19.11 \%$ oropharynx cancers. Among head and neck cancers, tongue cancer is most predominant (11.92\%) when compared to others. According to epidemiological investigations, $80-90 \%$ of all cancers are due to environmental factors among which, lifestyle related factors are the most predominant and preventable [10]. The major risk factors for cancer are tobacco, alcohol consumption. Tobacco consumption, either by way of chewing or smoking accounts for $50 \%$ of all cancers in men. Studies have shown that appropriate changes in lifestyle and social habits will reduce the mortality and morbidity caused by cancer [11]. This indicates the need for initiating primary and secondary prevention measures for control and prevention of cancers mainly head and neck cancers [12]. Breast cancer accounts for $17.86 \%$, and it is the most predominant one among women. Women above $45 \mathrm{yrs}$ are more prone to Cervical, Breast, Ovarian cancers and as the most predominant age group in our study was 41-60 years, these types of cancers more like other studies [13]. Gynecological cancers accounts for $11.05 \%$ including cervix, uterus and ovary. Cancers of gastro-intestinal tract $(10.61 \%)$ are also recorded in this population. This can be attributable to dietary habits.

The interesting findings of the present study are the Histopathological sub typing are varieties of reasons that can suspect a cancer, but the exact diagnosis is confirmed by histological examination of the cancerous cells from a biopsy or on postoperative histopathology. The tissue diagnosis indicates the type of cell that is proliferating, its histological grade. This information is useful for assessing the prognosis and choosing the modality of the treatment. We studied all the histopathological reports of our patients and found that squamous cell carcinomas $(56.69 \%)$ are the predominant type. However data of some patients was not available $(13.19 \%)$.Till today very few authors presented this histopathological data in the area of South India.

At time of diagnosis the stage of disease was recorded. But, data of some patients was not available (66.38\%). The most commonly applied staging system for solid tumors is the TNM classification. T represents tumor size ranging from $\mathrm{T}_{1}$ to $\mathrm{T}_{4}$, Carcinoma in situ as $\mathrm{T}_{\mathrm{is}}$. $\mathrm{N}$ is extent and quality of nodal involvement ranging from $\mathrm{N}_{0}$ to $\mathrm{N}_{3}$. M represents metastases, depending on their presence or absence M0 or M1 are denoted. Usually a numerical value is assigned to each letter to indicate the size or extent of disease. Majority of the cancers are 
classified based on extent of the disease as stages I to IV. Stage I is localized tumor, stages II, III are local and regional extension, and stage IV denotes the presence of distant metastases. The criteria for classifying disease extent vary from cancer to cancer [14]. Alternative alphabetical systems (stage A, B, C, or D) are also in clinical practice. Most of the patients in our study were in advanced stages like in Stage-III (6.38\%). Most of the patients were in advanced stages $(6.38 \%$ were in Stage-III).

Modality of treatment depends on the type and stage of the disease. Four primary modalities are employed in the approach to cancer treatment at our hospital are surgery, radiation, chemotherapy, and chemoirradiation. For most of the solid tumors diagnosed in the early stages, surgery remains the treatment of choice. Among all only $1 \%$ of patients underwent surgery, as most of the cancers were detected in advanced stages. Radiation therapy was first introduced in late 1800 s, since then it is the best management choice for cancer. In our study most of the patients $(60.42 \%)$ underwent radiation therapy. Chemotherapy enables systemic circulation and effective in treating primary and any metastatic disease. [15] It is given to $29.36 \%$ patients. Both chemotherapy and radiation therapy were given to $9.36 \%$ patients to combat the cancer.

The total duration of treatment varied from 1-18 months. Majority of the patients $(57.87 \%)$ were treated within six months of the admission. There is also a record of irregular follow -up in $1.27 \%$ and no follow-up in $31.91 \%$ patients. This is primarily because of the impact of emotional stress after cancer diagnosis and economical burden for treatment, especially in a developing country like India. The cancer experience has been found to induce fatigue, [16-18] which will influence the Quality of Life (QoL) in cancer patients and affect the follow -up. The follow-up of male and female patients is indicated in Table No.2.

\section{Table 2: Follow-up}

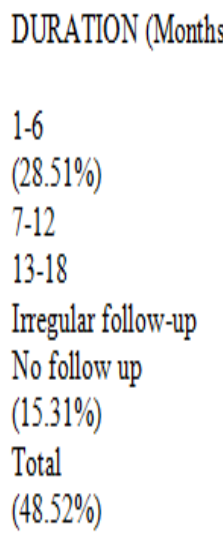

$\begin{array}{ccl}\text { TOTAL } & \text { MALE } & \\ \text { FEMALE } & & \\ 136(57.87 \%) & 69(29.36 \%) & 67 \\ & & \\ 20(8.51 \%) & 13(5.53 \%) & 7(2.97 \%) \\ 1(0.42 \%) & 0 & 1(0.42 \%) \\ 3(1.27 \%) & 0 & 3(1.27 \%) \\ 75(31.91 \%) & 39(16.59 \%) & 36 \\ & & \\ 235(100 \%) & 121(51.48 \%) & 114\end{array}$

\section{Conclusions}

This study explains in detail about the proportion of various cancers over this south Indian population. The quantities measure the changes in the occurrence and potential impact of primary prevention. This work provides a source of information, which help public health planners, administrators, healthcare team, and general public in the primary prevention and early detection of cancer. As older patients are more, constant and routine assessment of the Quality of Life (QoL) and factors that affect it may help. Most common type among male is cancer of tongue (1 1.91\%) and among female is breast cancer (17.8 7\%). Establishment of equitable, pain control and palliative care network like hospice can improve the follow-up. Avoiding preventable factors like tobacco and alcohol use, taking healthy diet, maintaining ideal Body mass index (BMI), avoiding sun ray exposure, vaccination against hepatitis B and human papilloma vaccine against carcinoma cervix, healthy practices and regular screening can prevent cancer.
[2]. Nandakumar A. National Cancer Registry Program, Indian Council of Medical Research,
[3]. Consolidated report of the population based cancer registries, New Delhi, India: 1990 -
[4]. 96.
[5]. Cancer Registry Abstract, Newslett. Natl. Cancer Registry Project India, 2001, 8.
[6]. Parkin DM, Whelan SL, Ferlay J, Raymond L \& Young. J. Cancer Incidence in five
[7]. continents . IARC Scientific Publications, Vol. VII (143), Lyon, 1997
[8]. SRS based abridged life tables 1990-94 and 1991-95. SRS Analytical Studies 1998, 1, 3.
[9]. N Malothu, U Veldandi, N Yellu, R Devarakonda \& N Yadala : Pharmacoepidemiology Of 
[10]. Cancer in Southern India. The Internet Journal of Epidemiology, 2010 Volume 8 Number

[11]. 1.

[12]. Litchfield MH. Environ Sci Pollut Res 1999; 6:175-182.

[13]. Popendorf W, Donham KJ. Agricultural hygiene. In: Patty's Industrial Hygiene and Toxicology, 1991, 4th ed, Vol 1, Pt A. New York: John Wiley \& Sons . pp. 721-761.

[14]. Lee PN. Smoking "attributable" mortality in India Some relevant considerations 1996-

[15]. 12: 12-18.

[16]. WHO, The World Health Report, Geneva, 1997.

[17]. Varghese C. Cancer prevention and control in India. Ministry of Health and Family

[18]. Welfare. Available at: http://mohfw.nic.in/pg56to67.pdf

[19]. urthy NS, Mathew A. Curr Sci 2004; 86(4): 518-524.

[20]. Roger Z, Anderson R, Cefalu C and Sidani M. Cancer screening guidelines American Family Physician 2001; 63(6): 20-23.

[21]. Fleming ID, et al., eds . AJCC Cancer Staging Manual, 6th Ed. New York: Springer-Verlag,

[22]. 2002:209-217.

[23]. Hardman JG, Limbird LE, Molinoff PB, eds. Goodman \& Gilman's The Pharmacologic

[24]. Basis of Therapeutics, 10th ed. New York: McGraw-Hill, 2001:1381-1388.

[25]. Servaes P, Verhagen C, Bleijenberg G. Eur J Cancer 2002; 38: 27-43.

[26]. Tavio M, Milan I, Tirelli U. Int J Oncology 2002; 21: 1093-1099.

[27]. Stasi R, Abriani L, Beccaglia P, Terzoli E, Amadori S. Cancer 2003; 98: 1786- 1801. 